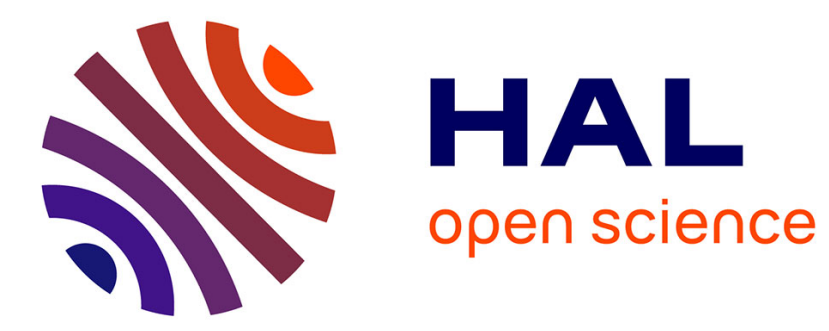

\title{
Krawtchouk polynomials and finite probability theory
}

Philip Feinsilver, René Schott

\section{To cite this version:}

Philip Feinsilver, René Schott. Krawtchouk polynomials and finite probability theory. [Research Report] RR-1744, INRIA. 1992. inria-00076984

\section{HAL Id: inria-00076984 https://hal.inria.fr/inria-00076984}

Submitted on 29 May 2006

HAL is a multi-disciplinary open access archive for the deposit and dissemination of scientific research documents, whether they are published or not. The documents may come from teaching and research institutions in France or abroad, or from public or private research centers.
L'archive ouverte pluridisciplinaire HAL, est destinée au dépôt et à la diffusion de documents scientifiques de niveau recherche, publiés ou non, émanant des établissements d'enseignement et de recherche français ou étrangers, des laboratoires publics ou privés. 


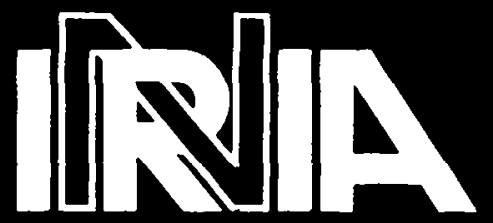

UNITÉ DE RECHERCHE INRIA-LORRAINE

Institut National de Recherche en Informatique et en Automatique

Domaine de Voluceau - Rocquencourt B.P.105

78153 Le Chesnay Cedex France Tél:(1) 39635511

\section{Rapports de Recherche}

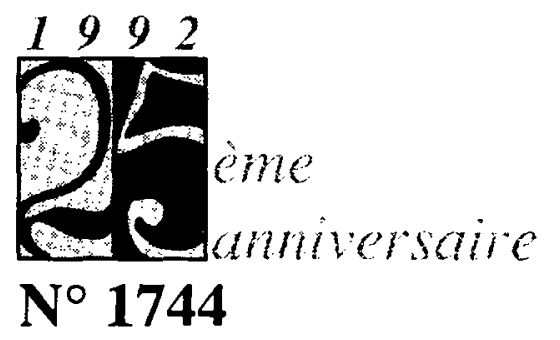

\section{Programme 2}

Calcul Symbolique, Programmation et Génie logiciel

\section{KRAWTCHOUK POLYNOMIALS AND FINITE PROBABILITY THEORY}

\section{Philip FEINSILVER}

René SCHOTT

Septembre 1992 


\title{
Krawtchouk Polynomials and Finite Probability Theory
}

\section{Polynômes de Krawtchouk et Probabilités sur un Univers fini}

Philip Feinsilver; $\quad$ René Schott `ं

\begin{abstract}
Some general remarks on random walks and martingales for finite probability distributions are presented. Orthogonal systems for the multinomial ditribution arise. In particular, a class of generalized Lrawtchouk polynomials is determined by a random walk generated by roots of unity. Relations with hypergeometric functions and some limit theorems are discussed.
\end{abstract}

\section{Résumé}

Nous présentons quelques propriétés concernant les marches aléatoires et les martingales quand les distributions sont définies sur des univers finis. Des systèmes orthogonaux pour la loi multinomiale sont mis en évidence. En particulier, une classe de polynomes de Lirawtchoul généralisés a pu être déterminée en considérant une marche alćat oire associée aux racines complexes de l'unité. Certains liens entre fonctions hypergéométriques et théorèmes limitess sont également explicit és.

"Departuent of Mathematics. Southern Illinois [iniversity. Carbondale. Illinols 62901 IIS.A

'CRIN, INRIA-Lorraine, Université de Nancy 1. 54.506 Vandoeuvre-les- Vancy, France' 


\title{
Krawtchouk Polynomials and Finite Probability Theory
}

\author{
P. Feinsilver ${ }^{1}$ and $R$. Schott ${ }^{2}$
}

\begin{abstract}
Some general remarks on random walks and martingales for finite probability distributions are presented. Orthogonal systems for the multinomial distribution arise. In particular, a class of generalized Krawtchouk polynomials is determined by a random walk generated by roots of unity. Relations with hypergeometric functions and some limit theorems are discussed.
\end{abstract}

\section{Introduction}

Krawtchouk polynomials $K_{\alpha}(x, N)$ are polynomials orthogonal with respect to a binomial distribution; for convenience we consider Bernoulli random variables taking values in \pm 1 with equal probability. They may be defined by the generating function

$$
G(v)=\sum_{\alpha=0}^{N} v^{\alpha} K_{\alpha}(x, N)=(1+v)^{(N+x) / 2}(1-v)^{(N-x) / 2}
$$

The generating function

$$
(1-v)^{y-a}(1-(1-R) v)^{-y}=\sum_{n=0}^{\infty} \frac{v^{n}}{n !}(a)_{n 2} F_{1}\left(\begin{array}{c}
-n, y \\
a
\end{array} \mid R\right)
$$

with $(a)_{n}=\Gamma(a+n) / \Gamma(a)$, yields the identification

$$
K_{\alpha}(x, N)=\left(\begin{array}{c}
N \\
\alpha
\end{array}\right){ }_{2} F_{1}\left(\begin{array}{c|c}
-\alpha,(x-N) / 2 & 2 \\
-N &
\end{array}\right)
$$

A theory for multivariate 'Bernoulli-type' polynomials is presented in [4]. Here we take a different look at generalized Krawtchouk polynomials.

One class arises from a random walk on the lattice $\mathrm{N}\left[1, \zeta, \zeta^{2}, \ldots, \zeta^{d-1}\right]$ where $\zeta=$ $e^{2 \pi i / d}$. There are evident connections with the finite Fourier transforn, see, e.g., [2], but a deeper study is yet to come. Some observations of interest will be made in the discussion in section V. It is quite likely that the classes of polynomials discussed here will prove useful in image processing, among other possible applications.

In section II we give the probabilistic approach to the binomial case. Section III provides a general approach for finite probability distributions. In section IV some related

1 Department of Mathematics, Southern Illinois University. Carbondale. Illinois 62901 USA

2 CRIN, INRIA-Lorraine, Université de Nancy I, B.P. 239, 54506-Vandoeure-lès-Nancy: France 
limit theorems are presented. Specialization to and special adjustments for the cyclic case comprise section $\mathrm{V}$ which completes the study.

Note. 1. Throughout, $N$ will denote 'time' - the number of steps taken in the random walk.

2. We use \langle\rangle to denote expected value, e.g. $\langle X\rangle$.

3. For brevity, we call Krawtchouk polynomials simply $\mathrm{K}$-polynomials.

\section{Binomial Case}

Consider a random walk on $\mathbf{Z}$, with equiprobable increments \pm 1 . We write $X_{j}, 1 \leq j \leq N$, for the corresponding Bernoulli variables.

The generating function

$$
G(v)=\prod\left(1+v X_{j}\right)=(1+v)^{(N+x) / 2}(1-v)^{(N-x) / 2}
$$

where $x=\sum X_{j}$ is the position after $N$ steps. As noted above, $G(v)=\sum v^{\alpha} I_{\alpha}(x, N)$. with Krawtchouk polynomials $K_{\alpha}^{-}$. One can take the viewpoint of 'quantum probability' and consider the $X_{j}$ as the spectrum of an operator $A$, an $N \times N$ matrix. Then the condition on $A$ is that $\lambda \in \operatorname{spectrum}(A) \Rightarrow \lambda \in\{-1,1\}$. And the variables of interest are the multiplicities. We have

$$
G(v)=\operatorname{det}(1+v \cdot A) \text {. }
$$

And the variables $x, N$ are $\operatorname{simply} \operatorname{tr} A, \operatorname{tr} A^{2}$. We thus have the principal observation that since the $X_{j}$ take two values, two variables suffice to specify the $K_{\alpha}$, which are seen to be elementary symmetric functions in the $X_{j}$. The variables $x, N$ are the corresponding power sums: $x=\sum X_{j}, N=\sum X_{j}^{2}$.

The generating function $G(v)$ as a function of $N$ is readily seen to be a martingale, as the $X_{j}$ are independent with mean zero. It is, in fact, the prototype exponential martingale (see [1], e.g.). The calculation

$$
\langle G(v) G(w)\rangle=\prod\left\langle 1+(v+w) X_{j}+v w X_{j}^{-2}\right\rangle=(1+v w)^{N}
$$

exhibits the orthogonality of the $K_{\alpha}$ nicely. So the $K_{\alpha}$ are important mainly for these two features:

1. They are the iterated integrals (sums) of the Bernoulli process.

2. They are orthogonal polynomials with respect to the binomial distribution.

\section{Finite Probability Distributions}

The probabilistic approach may be carried out for general finite probability spaces. Each increment $X$ takes $d$ possible values $\left\{\xi_{0}, \ldots, \xi_{\delta}\right\}$ with $P\left(X=\xi_{j}\right)=p_{j}, 0 \leq j \leq \delta$, where throughout we will use the convention $\delta=d-1$. Denote the mean and variance by $\mu$ and $\sigma^{2}$ as usual.

Take $N$ independent copies of $X^{-}: X_{j}, 1 \leq j \leq N$. Define the martingale

$$
G(v)=\prod_{j=1}^{N}\left(1+v\left(X_{j}-\mu\right)\right)
$$


We may switch to the multiplicities as variables. So set

$$
n_{j}=\sum_{k=1}^{N} \mathbf{1}_{\left\{X_{k}=\xi_{j}\right\}}
$$

the number of times the value $\xi_{j}$ is taken. We have

$$
G(v)=\prod_{j=0}^{\delta}\left(1+v\left(\xi_{j}-\mu\right)\right)^{n_{j}}=\sum_{\alpha=0}^{N} v^{\alpha} K_{\alpha}\left(n_{0}, \ldots, n_{\delta}\right)
$$

this last defining our generalized K-polynomials. One quickly gets

3.1 Proposition. Denoting the multi-index $\mathbf{n}=\left(n_{0}, \ldots, n_{\delta}\right)$ and by $\mathbf{e}_{\mathbf{j}}$ the standard basis on $\mathbf{Z}^{d}, K$-polynomials satisfy the recurrence

$$
K_{\alpha}\left(\mathbf{n}+\mathbf{e}_{\mathbf{j}}\right)=K_{\alpha}(\mathbf{n})+\left(\xi_{j}-\mu\right) K_{\alpha-1}(\mathbf{n})
$$

We also find by binomial expansion

\subsection{Proposition.}

$$
H_{\alpha}\left(n_{0}, \ldots, n_{\delta}\right)=\sum_{|\mathbf{k}|=\alpha} \prod\left(\begin{array}{l}
n_{j} \\
k_{j}
\end{array}\right)\left(\xi_{j}-\mu\right)^{k_{j}}
$$

where $\mid \mathbf{k}\}=k_{0}+\cdots+k_{6}$.

An interesting connection with the multivariate theory comes in when we consider equations (1.2), (1.3). The Lauricella polynomials $F_{B}$ are defined by

$$
F_{B}\left(\begin{array}{c|c}
-\mathbf{r}, \mathbf{b} & \mathbf{s} \\
t &
\end{array}\right)=\sum_{\mathbf{k} \in \mathbf{N}^{6}} \frac{(-\mathbf{r})_{\mathbf{k}}(\mathbf{b})_{\mathbf{k}}}{(t)_{|\mathbf{k}|} \mathbf{k} !} \mathbf{s}^{\mathbf{k}}
$$

with, e.g., $\mathbf{r}=\left(r_{1}, \ldots, r_{\delta}\right),(\mathbf{r})_{\mathbf{k}}=\left(r_{1}\right)_{k_{1}}\left(r_{2}\right)_{k_{2}} \cdots\left(r_{\delta}\right)_{k_{\delta}}$ for multi-index $\mathbf{k}$, also $\mathbf{s}^{\mathbf{k}}=$ $s_{1}^{k_{1}} \cdots s_{\delta}^{k_{6}}$, and $\mathbf{k} !=k_{1} ! \cdots k_{\delta} !$. Note that $t$ is a single variable. The generating function of interest here is

$$
\left(1-\sum v_{i}\right)^{\sum b_{j}-t} \prod_{j}\left(1-\sum v_{i}+s_{j} v_{j}\right)^{-b_{j}}=\sum \frac{\mathbf{v}^{\mathbf{r}}(t)_{|\mathbf{r}|}}{\mathbf{r} !} F_{B}\left(\begin{array}{c}
-\mathbf{r}, \mathbf{b} \\
t
\end{array} \mid \mathbf{s}\right)
$$

a multivariate version of (1.2).

3.3 Proposition. If $\xi_{0}=0$, then

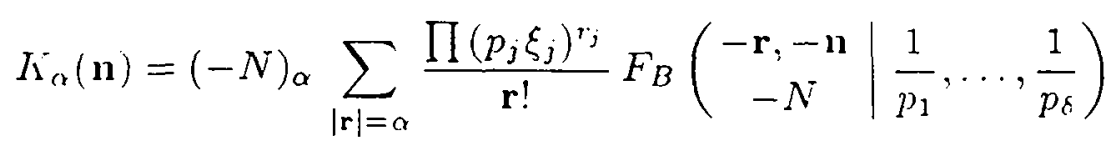


Proof: Let $v_{j}=v p_{j} \xi_{j}, b_{j}=-n_{j}, t=-N, s_{j}=p_{j}^{-1}$ in (3.7), for $1 \leq j \leq \delta$. Note that $\sum v_{j}=v \mu, \sum b_{j}-t=N-\left(n_{1}+\cdots+n_{\delta}\right)=n_{0}$.

As for the binomial case, we may use the power sum variables, e.g. for centered increments,

$$
x_{i}=\sum_{j=1}^{N}\left(X_{j}-\mu\right)^{i}=\sum_{j=0}^{\delta} n_{j}\left(\xi_{j}-\mu\right)^{i}
$$

to express the functions $K_{\alpha}$. (This will be useful in the cyclic case, section V below.)

Orthogonality follows similar to the binomial case.

3.4 Proposition. The $K$-polynomials $K_{\alpha}\left(n_{0}, \ldots, n_{\delta}\right)$ are orthogonal with respect to the induced multinomial distribution. In fact,

$$
\left\langle K_{\alpha} K_{\beta}\right\rangle=\delta_{\alpha \beta} \sigma^{2 a}\left(\begin{array}{l}
N \\
\alpha
\end{array}\right)
$$

Proof:

$$
\begin{aligned}
\langle G(v) G(w)\rangle & =\sum\left(\begin{array}{c}
N \\
n_{0}, \ldots, n_{\varepsilon}
\end{array}\right) p_{0}^{n_{0}} \cdots p_{\delta}^{n_{\delta}} \prod\left(1+(v+w)\left(\xi_{j}-\mu\right)+v w\left(\xi_{j}-\mu\right)^{2}\right)^{n_{j}} \\
& =\left(\sum\left(p_{j}+(v+w) p_{j}\left(\xi_{j}-\mu\right)+v w p_{j}\left(\xi_{j}-\mu\right)^{2}\right)\right)^{N}
\end{aligned}
$$

Thus, $\langle G(v) G(v)\rangle=\left(1+v w \sigma^{2}\right)^{N}$. This shows orthogonality and yields the squared norms as well.

\section{Limit Theorems}

Limit theorems for products $\prod\left(1+v X_{j}\right)$ are given by [1] for $X_{j}$ discrete increments of a process. In cases discussed there, the limits yield iterated integrals of the process, as may be expected (see [3, Ch. VII]).

Here (and in section V) we look at limit theorems based on the power sum functions. First, define the normalized values $\beta_{j}=\left(\xi_{j}-\mu\right) / \sigma$. Then the central limit theorem says that

$$
\frac{1}{\sigma \sqrt{N}} \sum_{j=1}^{N}\left(X_{j}-\mu\right)=\frac{1}{\sqrt{N}} \sum_{j=0}^{\delta} n_{j} \beta_{j}
$$

converges in distribution to a standard Gaussian as $N \rightarrow \infty$. We look at the power sums of the normalized variables:

$$
\sum\left(\frac{X_{j}-\mu}{\sigma \sqrt{N}}\right)^{i}=N^{-i / 2} \sum n_{j} \beta_{j}^{i}
$$

4.1 Theorem. Let $Y_{j}$ satisfy $n_{j}=p_{j} N+Y_{j} \sqrt{N}$. Then, as $N \rightarrow \infty, \sum_{j=0}^{\gamma} Y_{j} \beta_{j}$ converges to a standard Gaussian. 
Proof: We have, recalling that $\sum p_{j} \beta_{j}=0$,

$$
\frac{1}{\sqrt{N}} \sum n_{j} \beta_{j}=\frac{1}{\sqrt{N}} \sum\left(p_{j}+Y_{j} \sqrt{N}\right) \beta_{j}=\sum Y_{j} \beta_{j}
$$

and the result follows from the central limit theorem.

4.2 Theorem. The martingale for the normalized variables

$$
G(v)=\prod_{j=1}^{N}\left(1+v \frac{X_{j}-\mu}{\sigma \sqrt{N}}\right)
$$

converges to the Brownian martingale at time 1,

$$
G(v) \rightarrow e^{v Y-v^{2} / 2}
$$

where $Y$ is the standard Gaussian denoting the limit of the normalized sums.

Proof: Write

$$
\begin{aligned}
\prod_{j=1}^{N}\left(1+v \frac{X_{j}-\mu}{\sigma \sqrt{N}}\right) & =\prod_{j=0}^{\delta}\left(1+v \beta_{j} / \sqrt{N}\right)^{n_{j}} \\
& =\prod_{\exp \left(n_{j} \log \left(1+v \beta_{j} / \sqrt{N}\right)\right)} \\
& =\exp \left(\sum_{i=1}^{\infty}(-1)^{i-1} \frac{v^{i}}{i} S_{i}\right)
\end{aligned}
$$

where $S_{i}$ are the scaled power sums $N^{-i / 2} \sum n_{j} \beta_{j}^{i}$. Theorem (4.1) deals with $i=1$. For $i=2$ we have, with $n_{j}=p_{j} N+Y_{j} \sqrt{N}$,

$$
N^{-1} \sum n_{j} \beta_{j}^{2}=\sum p_{j} \beta_{j}^{2}+N^{-1 / 2} \sum Y_{j} \beta_{j}^{2}=1+o(1)
$$

since the $\beta$ 's are scaled to variance 1 . For $i \geq 3$, we have

$$
N^{1-i / 2} \sum p_{j} \beta_{j}^{i}+N^{(1-i) / 2} \sum Y_{j} \beta_{j}^{i} \rightarrow 0
$$

as $N \rightarrow \infty$. Denoting the limit of $\sum Y_{j} \beta_{j}$ by $Y$ the result follows.

We conclude that the corresponding K-polynomials converge to Hermite polynomials in the variable $Y$.

\section{Cyclic K-polynomials}

Now we consider a random walk in $\mathbf{C}$, with increments $X$ taking values in the $d$ th roots of unity: $1, \zeta, \zeta^{2}, \ldots, \zeta^{\delta}$, with $\zeta=e^{2 \pi i / d}$, and $\delta=d-1$ as above. For simplicity we discuss the isotropic case, all values occurring with equal probability $1 / d$. 
The distribution of $x=$ position after $N$ steps is an interesting problem in number theory. Namely, to count how many 'paths' lead to the same position $x$. Here we make some remarks concerning this problem. Again, let $n_{j}$ denote the multiplicities of occurrences of the $\zeta^{j}$. We have the power sum variables:

$$
\begin{aligned}
& x_{0}=n_{0}+n_{1}+\ldots=N \\
& x_{1}=n_{0}+n_{1} \zeta+\ldots=\sum n_{j} \zeta^{j}=x \\
& x_{2}=n_{0}+n_{1} \zeta^{2}+\ldots=\sum n_{j} \zeta^{2 j} \\
& \vdots \\
& x_{k}=n_{0}+n_{1} \zeta^{k}+\ldots=\sum n_{j} \zeta^{k j}
\end{aligned}
$$

Notice that the $x_{k}$ are the finite Fourier transform of the variables $\left(n_{0}, \ldots, n_{\delta}\right)$. So herc we can conveniently go back and forth between the two sets of variables.

From algebraic number theory, see [5, p.265ff.], it is known that for $d=$ a prime power, $\phi(d)$ denoting Euler's function, the powers $1, \zeta, \zeta^{2}, \ldots, \zeta^{\phi(d)-1}$ form a basis for the Z-module spanned by the $\zeta^{j}, 0 \leq j \leq \delta$. I.e. each sum of the form $\sum n_{j} \zeta^{j}$ has a unique expression as a sum involving $\zeta^{j}$ for $j<\phi(d)$. The problem is that we have to count how many general sums, involving all the $\zeta^{j}$, reduce to the same canonical form, involving only: $\zeta^{j}, j<\phi(d)$. From the point of view of Fourier analysis and probablility theory this has the flavor of finite prediction theory and requires a separate study.

Here we note that if $d$ is a prime, then $1, \zeta, \zeta^{2}, \ldots, \zeta^{d-2}$ form a $\mathbf{Z}$-basis. And we have the elementary relation $\sum \zeta^{j}=0$. Thus,

5.1 Proposition. Let $d$ be prime. Given $x_{0}=N$ and $x_{1}=\sum n_{j} \zeta^{j}$, the $n_{j}$ are uniquely: determined.

Proof: In $x_{1}$, substitute $\zeta^{\varepsilon}=-1-\zeta-\cdots-\zeta^{\delta-1}$ yielding

$$
x_{1}=\left(n_{0}-n_{\delta}\right)+\zeta\left(n_{1}-n_{\delta}\right)+\cdots+\zeta^{\delta-1}\left(n_{\delta-1}-n_{\delta}\right)
$$

By the result quoted above, the numbers $n_{j}-n_{\delta}, 0 \leq j<\delta$ are uniquely determined. Thus, their sum, call it $\nu=n_{0}+\cdots+n_{\delta-1}-\delta n_{\delta}$ is known, thence $d n_{\delta}=n_{0}+\cdots+n_{\delta}-\nu=N-\nu$ is determined and hence the $n_{j}, 0 \leq j<\delta$ as well.

Now we look at the K-polynomials for the cyclic case. The generating function is

$$
G(v)=\prod_{j=0}^{\delta}\left(1+v \zeta^{j}\right)^{n_{j}}=\sum_{\alpha=0}^{N} v^{\alpha} K_{\alpha}
$$

They satisfy the recurrence, cf. Proposition (3.4),

$$
\Lambda_{\alpha}\left(\mathbf{n}+\mathbf{e}_{\mathbf{j}}\right)=K_{\alpha}(\mathbf{n})+\zeta^{j} \Lambda_{\alpha-1}(\mathbf{n})
$$


The binomial expansion gives

$$
K_{\alpha}\left(n_{0}, \ldots, n_{\delta}\right)=\sum_{|k|=\alpha} \prod\left(\begin{array}{l}
n_{j} \\
k_{j}
\end{array}\right) \zeta^{j k_{j}}
$$

The $F_{B}$ representation is found as follows. In the generating function $(3.7)$, let $v_{j}=$ $v \zeta^{j}, 1 \leq j \leq \delta$. With $b_{j}=-n_{j}, 1 \leq j \leq \delta, t=-N$, we have

$$
\sum_{j=1}^{\delta} v_{j}=-v \quad \text { and } \quad \sum_{j=1}^{\delta} b_{j}=n_{0}-N
$$

With $s_{j}=1-\bar{\zeta}^{j}$, writing $\bar{\zeta}=\zeta^{-1}$ as usual,

$$
1-\sum v_{j}+s_{j} v_{j}=1+v+\left(1-\bar{\zeta}^{j}\right) v \zeta^{j}=1+v \zeta^{j}
$$

for $1 \leq j \leq<d$. Thus, as in Proposition (3.3),

$$
K_{\alpha}=(-N)_{\alpha} \sum_{|\mathbf{r}|=\alpha} \frac{\zeta^{\sum j r_{j}}}{\mathbf{r} !} F_{B}\left(\begin{array}{c}
-\mathbf{r},-\mathbf{n} \\
-N
\end{array} \mid 1-\bar{\zeta}, \ldots, 1-\bar{\zeta}^{\delta}\right)
$$

Finally we have the limit result

5.2 Theorem. In $G(v)$, scale $v \rightarrow v n^{-1 / d}, x_{0} \rightarrow x_{0} n$ and, for $1 \leq k \leq \delta, x_{k} \rightarrow$ $x_{k} n^{(d, k) / d}$, where $(d, k)=$ greatest common divisor of $d$ and $k$. Then

$$
G(v) \rightarrow \exp \left(\sum_{k \mid d}(-1)^{k-1} v^{k} x_{k} / k\right)
$$

as $n \rightarrow \infty$.

Proof: Fourier inversion says that $n_{j}=d^{-1} \sum x_{k} \bar{\zeta}^{j k}$. I.e.

$$
G(v)=\prod_{j}\left(1+v \zeta^{j}\right)^{\sum \bar{\zeta}^{j k} x_{k} / d}=\prod_{j, k}\left(1+v \zeta^{j}\right)^{\bar{\zeta} j k} x_{k} / d
$$

For fixed $k$, let $(d, k)=g$. Then, for $0 \leq j<d$, we can write $j=l d / g+r$, with $r<d / g$. Thus,

$$
j k \equiv l(d k / g)+r k \equiv r k \quad(\bmod d)
$$

So

$$
\begin{aligned}
\prod_{j=0}^{\delta}\left(1+v \zeta^{j}\right)^{\zeta^{j k} x_{k} / d} & =\prod_{r=0}^{(d / g)-1} \prod_{l=0}^{g-1}\left(1+v \zeta^{r+l d / g}\right)^{\zeta^{r k} x_{k} / d} \\
& =\prod_{r=0}^{(d / g)-1}\left(1+\left(v \zeta^{r}\right)^{g}(-1)^{g-1}\right)^{j \zeta x_{k} / d}
\end{aligned}
$$


since

$$
\prod_{l=0}^{g-1}\left(1+y \zeta^{l d / g}\right)=1+y^{g} \zeta^{(d / g)(g(g-1) / 2)}=1+y^{g}(-1)^{g-1}
$$

With the scalings indicated, $x_{k} \rightarrow x_{k} n^{g / d}, v \rightarrow v n^{-1 / d}$, each factor of the product in (5.12) will converge to expressions of the form

$$
\exp \left(v^{g} \zeta^{r g}(-1)^{g-1} \bar{\zeta}^{r k} x_{k} / d\right)
$$

Taking the product over $0 \leq r<d / g$, we have

$$
\sum_{r=0}^{(d / g)-1} \zeta^{r(g-k)}=\frac{\bar{\zeta}^{k \cdot d / g}-1}{\zeta^{g-k}-1}=0
$$

if $g \neq k$, and $=d / g$ for $g=k$, i.e. when $k \mid d$.

It may be of interest to compare with [6].

\section{Concluding Remarks}

It looks like an analysis for general abelian groups based on similar ideas should be feasible. It is interesting to recall that the $\mathrm{K}$-polynomials in the binomial case provide representation spaces for $s u(2)$ and are thus connected with spherical harmonics. Similar connections/interpretations for general K-polynomials would be quite interesting to find.

Acknowledgement. We are grateful to Jerzy Kocik for stimulating conversations. 


\section{References}

1. F. Avram: Weak convergence of the variations, iterated integrals and Doleans-Dade exponentials of sequences of semimartingales, Ann. Prob., 16, 1988, 246-250.

2. Ph. Feinsilver and R. Schott: Orthogonal polynomial expansions via Fourier transform, preprint, 1990.

3. Ph. Feinsilver: Special functions, probability semigroups, and Hamiltonian fows, Lecture Notes 696, Springer-Verlag, 1978.

4. Ph. Feinsilver: Bernoulli systems in several variables, Lecture Notes 1064, 1984, $86-98$.

5. P. Ribenboim: Algebraic numbers, Wiley-Interscience, 1972.

6. W. von Waldenfels: Noncommutative central limit theorems, Lecture Notes $\mathbf{1 2 1 0}$, 1986, 174-202. 
ISSN 0249-6399 